Article

\title{
Dynamic Residential Energy Management for Real-Time Pricing
}

\author{
Leehter Yao ${ }^{1, * \mathbb{D}}$, Fazida Hanim Hashim ${ }^{2}$ and Chien-Chi Lai ${ }^{1}$ \\ 1 Department of Electrical Engineering, National Taipei University of Technology, Taipei 10608, Taiwan; \\ b041632@gmail.com \\ 2 Faculty of Engineering and Built Environment, National University of Malaysia, Selangor 43600, Malaysia; \\ fazida@ukm.edu.my \\ * Correspondence: 1tyao@ntut.edu.tw; Tel.: +886-2-27712171 (ext. 2174)
}

Received: 4 May 2020; Accepted: 12 May 2020; Published: 18 May 2020

\begin{abstract}
A home energy management system (HEMS) was designed in this paper for a smart home that uses integrated energy resources such as power from the grid, solar power generated from photovoltaic (PV) panels, and power from an energy storage system (ESS). A fuzzy controller is proposed for the HEMS to optimally manage the integrated power of the smart home. The fuzzy controller is designed to control the power rectifier for regulating the AC power in response to the variations in the residential electric load, solar power from PV panels, power of the ESS, and the real-time electricity prices. A self-learning scheme is designed for the proposed fuzzy controller to adapt with short-term and seasonal climatic changes and residential load variations. A parsimonious parameterization scheme for both the antecedent and consequent parts of the fuzzy rule base is utilized so that the self-learning scheme of the fuzzy controller is computationally efficient.
\end{abstract}

Keywords: fuzzy controller; home energy management system; genetic algorithm; photovoltaic panel; real-time price

\section{Introduction}

Recent technological advances in smart grids, communication technologies, and renewable energy sources (RESs) have led to significant changes in the management of energy resources. Due to the increasing residential energy demand, rising energy costs, and environmental concerns, appropriate management of the energy from smart grids and RES is crucial. A home energy management system (HEMS) provides the necessary tool for appropriate energy management, while giving considerations to pertaining factors, such as connectivity to energy storage systems (ESS), participation in demand response (DR) programs, minimization of total user electricity cost, and environmental issues [1].

However, this technological opportunity comes with several challenges. The integration of the ESS with smart appliances and the participation in DR programs is a complex integration that requires active and governed participation of users at the residential end. Currently, the participation in DR programs is seemingly low due to user's lack of knowledge in responding to DR programs and lack of appropriate energy management schemes in the HEMS [2,3]. By using wired or wireless communication technologies along with the HEMS, users can monitor and control the residential energy usage in a sophisticated and accurate manner [4]. This technological advancement not only allows users to participate in DR programs, it also improve energy management efficiency, users' comfort and communication security.

One of the most attractive functions of the HEMS is that it saves electricity cost in response to the dynamic electricity prices without affecting users' comfort. The users participating in DR programs uses the HEMS to schedule the use of their residential controllable appliances to the time at which 
electricity prices are lower to reduce their electricity cost [5-8]. Conversely, the HEMS can also manage the connected energy supplying systems such as PV panels $[9,10]$ and ESSs $[11,12]$ to provide the necessary energy for powering appliances instead of using only the power from grids.

In order to reflect the fluctuated wholesale electricity prices to end customers, time-differentiated pricing models such as real-time pricing (RTP), critical-peak pricing (CPP), day-ahead pricing (DAP), time-of-use pricing (TOUP), etc., have been adopted [13] in different DR programs. The end customers tend to adjust their electricity consumption in response to these time-differentiated prices. Among these pricing models, RTP is the most effective model in terms of reducing the peak reduction and load curve flattening $[14,15]$. It is common for the HEMS to optimize the appliance operation scheduling under the time-differentiated electricity pricing models. The electricity prices in the next $24 \mathrm{~h}$ for DAP or TOUP are acquired by the end customers. The operation scheduling of most residential appliances can thus be optimized based on the received price profile using HEMS. However, if the RTP is adopted, the price signals for the next $24 \mathrm{~h}$ or next long enough period of time are no longer available because the end customer can only receive the prices in real-time. Some simple linear schemes such as ARMA model [16] is usually utilized to predict the electricity prices. Other complex machine learning approaches such as support vector machine [17], neural networks [18], or genetic algorithm [19] are also applied to the price prediction in order to increase prediction accuracy. The electricity prices are predicted from the current step to the end of optimization horizon for the appliance scheduling optimization in HEMS. The scheduling optimization accuracy can be reasonably attained under the conditions that enough number of price data are collected for the price prediction, and the fluctuations of future prices are within a reasonable range. The intervals updating real-time prices usually range from $10 \mathrm{~min}$ to $1 \mathrm{~h}$. The appliances scheduling and energy management for HEMS with RTP relies on the price prediction scheme and prediction horizon. Different price prediction schemes are with different statistical variance and bias.

In this paper, an efficient HEMS-based residential energy management approach is proposed for RTP but without price prediction. A real-time approach capable of calculating the amount of power drawn from the grid while managing all energy resources and minimizing electricity cost is a viable solution of energy management for the RTP without price prediction. A fuzzy controller is proposed for the HEMS to control the rectifier that dynamically determines the power drawn from the grid. The fuzzy controller is designed with a simple computational structure so that the amount of power from the grid required to balance the overall residential energy resources is calculated in real time. In other words, the power drawn from the grid can be determined instantaneously in response to residential power consumption, solar energy generation from PV panels, the status of charging (SOC) of the ESS, and the real-time electricity prices. The proposed fuzzy controller dynamically determines the power drawn from the grid to reduce the electricity cost of smart homes.

Both load scheduling and energy management allow the HEMS to save energy cost and participate in DR programs under dynamic electricity prices. In [20-23], a household's electricity cost was minimized using the HEMS to automate the energy usage in smart homes in response to dynamic prices. The partially observable Markov decision process optimization approach was utilized in [20] to optimize appliance scheduling. In [21], a stochastic dynamic programming was applied to optimize a set of appliances to be controlled while considering the uncertainty in real-time prices. Dynamic programming and mixed integer linear programming were utilized in [22] and [23], respectively, to optimize appliance scheduling for time-varying electricity prices. The HEMS considers the aforementioned factors while optimizing load scheduling and energy management in order not to affect residential users' comfort or convenience of using appliances [24-26]. In [24], users' discomfort levels were defined in terms of the volume of energy curtailed by the HEMS. The quality of experience based on the knowledge of the annoyance suffered by users was defined in [25] as the discomfort level along with the load scheduling optimization. The inverse of the total waiting time of appliances was utilized as the comfort level in [26]. As air conditioners and heaters are one of most power consuming appliances, they are categorized into the list of controlled appliances by the HEMS [27-29]. However, 
directly calculating the power consumption of air conditioners and heaters is a challenging work. In $[27,28]$, the mathematic models to determine indoor temperatures associated with air conditioners and heaters are utilized for the scheduling optimization.

Along with the appliance scheduling optimization, the power that is drawn from the grid can also be optimized using an optimization tool such as mixed-integer linear programming (MILP) [30,31], meta-heuristic approach including genetic algorithm (GA) [32,33], and particle swarm optimization (PSO) [34,35]. It is common for smart homes to integrate renewable energy resources such as solar energy, wind energy, and the energy obtained from the power grid. To overcome the uncertainty of using renewable energy intermittently, stochastic programming was utilized as the optimization approach in the HEMS $[36,37]$. However, optimization is a computationally expensive approach and occupies most of the computational resource and time of the central processing unit (CPU) in the HEMS. In addition to scheduling optimization, the HEMS is designed to conduct monitoring and control functions for all connected meters, devices, appliances, etc.

A self-learning scheme is designed in the proposed fuzzy controller to allow the HEMS to adapt to the variations of the residential power consumption, historical price data, as well as short-term climatic changes. The fuzzy rule base needs to be refined from time to time through the self-learning. Numerous self-learning approaches have been explored for fuzzy controllers. In [38] and [39], a neural fuzzy controller was proposed using the gradient descent approach. The self-learning approach for adaptive fuzzy controller proposed in [40] and [41] requires a mathematical model of the system to be controlled although sometimes it is not easy to be accurately measured. Other meta-heuristic optimization approaches such as GA [42,43], ant colony optimization [44,45], and PSO [46,47] have also been applied to fuzzy controller learning. However, low computational power CPUs are generally designed in the HEMS for cost reduction. The self-learning approach proposed in this paper needs to be computationally simple but efficient. A fuzzy controller with orthogonal modulated triangular membership functions (MTMF) [48,49] is designed in this paper. The MTMF is a parsimonious parameterization scheme for membership functions that only requires two parameters to define the membership functions for every input in the antecedent part of the entire fuzzy rule base. An efficient approach for designing the membership functions in the consequent part of the fuzzy rule base will also be proposed in this paper. The computational efficiency of self-learning scheme for the fuzzy controller can be greatly improved. The GA is applied to optimize the fuzzy controller in this paper. With a simplified organization of the fuzzy rule base, very limited time is required to learn the fuzzy controller in the HEMS with lower computational power of the CPU. The proposed fuzzy controller is a fast online controller that continuously controls the rectifier output. However, the self-learning of the fuzzy controller is an offline scheme and yet is computationally efficient due to the parsimonious parameterization design of fuzzy rule base. The same idea with on-line fuzzy controller and computationally efficient off-line self-learning can be applied to the systems such as data centers [50] or tele-operation of robots [51,52] that requires complex real-time control.

The technical novelty and main contribution of this paper are summarized as follows:

1. A HEMS integrating energy resources from the grid, ESS, and PV panels is designed and implemented for RTP without price prediction. A self-learning fuzzy controller is designed in the HEMS dynamically controlling the rectifier output to reduce the electricity cost. To the best of our knowledge, no similar studies addressing this issue have been proposed in literature.

2. To reduce the computational effort required for self-learning, a novel membership function known as a MTMF is proposed to parameterize the fuzzy rule base so that the number of parameters required to be learned for the entire fuzzy rule base is greatly reduced.

3. The GA is applied to the learning of the fuzzy rule base to minimize the electricity cost paid to the utility company. Although the GA-based learning is conducted offline, it does not require too much time to obtain convergent solutions because the number of parameters to be learned and the search range of every parameter have been greatly reduced. 
4. Although GA is a random-search based learning approach, the search range of every parameter is mathematically analyzed before learning. The search range of every parameter is pinpointed and focused to a small range.

The rest of this paper is organized as follows. Section 2 introduces the structure of the HEMS investigated in this paper. Section 3 describes the fuzzy rule base and analyzes the characteristics of the proposed fuzzy controller with MTMF. Section 4 introduces the self-learning scheme of the proposed fuzzy controller. The experiments conducted using the proposed fuzzy controller are presented in Section 5, and the conclusions are drawn in Section 6.

\section{Home Energy Management System}

The structure of the power system with the home area network (HAN) for a smart home is shown in Figure 1 where all power sources are controlled and monitored by the HEMS through the HAN. All power sources are integrated and connected to the DC bus and the DC power is converted to the AC power through an inverter. All residential loads and converted AC power are connected to the AC bus. The DC power mainly comes from the PV panels, the lead-acid battery based ESS, and the power from the grid that is converted to DC through a rectifier. Since the output resistance of the ESS is much smaller than that of other power sources in Figure 1, the ESS discharges as soon as the voltage on the DC bus is below a preset threshold. Therefore, the ESS and PV panel are usually installed together because the ESS can compensate for the sudden voltage drops on the DC bus due to an unexpected decrease in the DC power or abrupt residential load increase. To compensate for the unexpected insufficiency of renewable DC energy sources, AC power from the grid is fed into the DC bus through a rectifier. The amount and scheduling of AC power is determined by the HEMS in response to the variations in the solar energy generated from PV panels, load demands at home, SOC of the ESS, and the RTP.

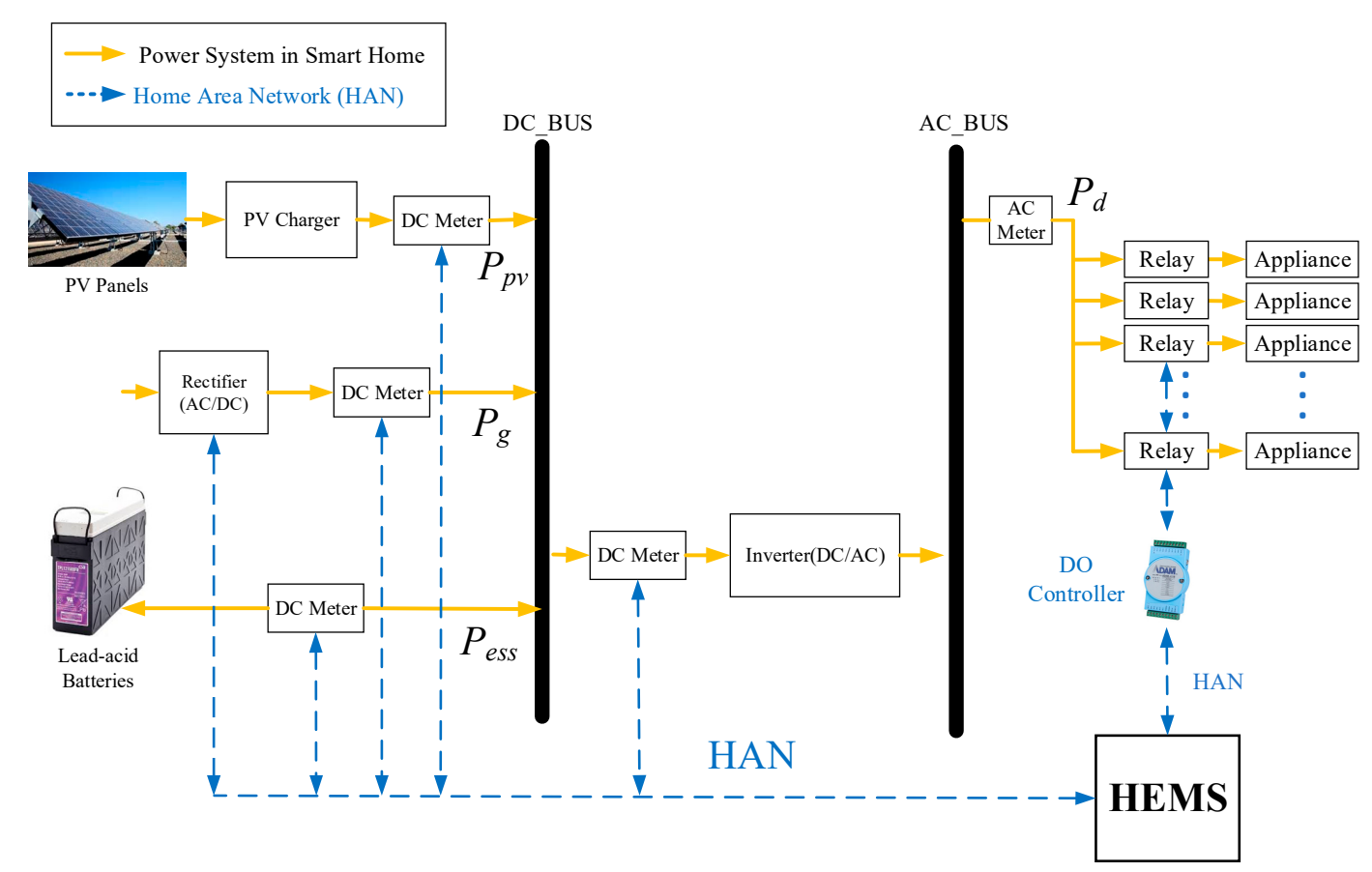

Figure 1. Structure of a power system monitored and controlled by home energy management system (HEMS).

A fuzzy controller controlling the rectifier output is designed in the HEMS. The fuzzy controller is a tailor-made controller because both the solar energy generated from PV panels and load demands vary for every smart home. It takes time to collect the information of the solar energy generated by PV panels 
and the load demands for every home. Engineers need to put in great efforts to fine tune the fuzzy controller for the HEMS in every home. A fast and effective self-learning fuzzy controller is designed to overcome this practical environmental uncertainties for HEMS installation at different homes. The fuzzy controller in every newly implemented HEMS comes with an initial parameter setting that results in reasonable energy management performance. After gaining reasonable amount of data such as daily solar energy profile data, daily load demand profiles, and historical data of RTP for a certain number days, the HEMS conducts self-tuning for the parameters in the fuzzy controller. The self-learning of the fuzzy controller can be conducted periodically or in response to environmental changes.

The fuzzy controller in the HEMS controls the rectifier output at every sampling interval $T_{s}$. Denote the power sources $P_{p v}^{j}, P_{g}^{j}$, and $P_{e S s}^{j}$ as the output power of the PV panels, rectifier, and ESS, respectively, $P_{d}^{j}$ as the load demand, at the $j$-th time step. The power sources and the load demand are balanced as follows:

$$
P_{d}^{j}=P_{p v}^{j}+P_{g}^{j}+P_{e s s}^{j}
$$

Denote $P_{S}^{j}$ as the net load demand showing if the solar power is sufficient to provide the household load demand, that is, $P_{s}^{j}=P_{d}^{j}-P_{p v}^{j}$. Let $\gamma^{j}$ and $\sigma^{j}$ be the SOC of the ESS and the RTP at the $j$-th time step, respectively. The power from the grid $P_{g}^{j}$ at every $j$-th time step depends on the net load demand $P_{s}^{j}$, the SOC of ESS $\gamma^{j}$, and the RTP $\sigma^{j}$ at the $j$-th time step. Denote $\mathbb{F}(\cdot)$ as the fuzzy controller in the HEMS, then the rectified power from the grid at the $j$-th time step can be defined as:

$$
P_{g}^{j}=\left\{\begin{array}{c}
\mathbb{F}\left(P_{s}^{j}, \gamma^{j}, \sigma^{j}\right), \text { if } P_{s}^{j}>0 \\
0, \text { otherwise. }
\end{array}\right.
$$

It is shown in Equation (2) that the HEMS does not need power from the grid if the solar energy is sufficient to self-support the load demand.

If $P_{g}^{j}$ is more than $P_{s}^{j}$, the additional power can be used to charge the ESS as long as the SOC $<1$. Conversely, the ESS discharges to the DC bus maintaining the load balance stated in Equation (1) if $P_{g}^{j}$ is not sufficient to support $P_{S}^{j}$ due to a sudden increase in the load demand $P_{d}^{j}$ or a sudden decrease in the solar power. The power charging ESS from the DC bus or the power ESS discharges to the DC bus is defined as follows:

$$
P_{e s s}^{j}=P_{g}^{j}-P_{s}^{j} .
$$

The ESS is in the charging mode if $P_{\text {ess }}^{j}>0$ and is in the discharging mode otherwise. The SOC of the ESS can be updated at every $j$-th time step as follows:

$$
\gamma^{j}=\gamma^{j-1}+\eta \frac{P_{e s s}^{j} T_{s}}{E^{\text {cap }}}
$$

where $T_{S}$ is the sampling interval, and $E^{\text {cap }}$ is the capacity of the ESS. Moreover, the efficiency constant $\eta=b_{c}$ if the ESS is in the charging mode, $\eta=1 / b_{d}$ if the ESS is in the discharging mode.

\section{Fuzzy Controller with Orthogonal Modulated Membership Functions}

The fuzzy controller $\mathbb{F}(\cdot)$ in Equation (2) is designed in this section. The proposed fuzzy controller is to control the rectifier in real-time and to continuously determine the rectified power into the smart home from the grid. The design also puts the need of off-line self-learning for the fuzzy controller into consideration. A computationally efficient parsimonious parameterization scheme is designed in the fuzzy controller. The orthogonal MTMFs provide excellent parsimonious parameterization effect. The antecedent parts of the fuzzy controller proposed are defined with MTMFs. 


\subsection{Fuzzy Controller Design}

A fuzzy controller is utilized to control the rectified AC power from the grid as in Equation (2). Assume that $A_{1}^{i_{1}}, A_{2}^{i_{2}}$, and $A_{3}^{i_{3}}$ are the antecedent fuzzy sets for the inputs $P_{s^{\prime}}^{j} \gamma^{j}$, and $\sigma^{j}$, respectively, and $g^{i_{1} i_{2} i_{3}}$ is the consequent fuzzy singleton for the output $P_{g}^{j}$ at every $j$-th time step. The proposed fuzzy controller can be described by the following fuzzy rules:

$$
\text { If } P_{s}^{j} \text { is } A_{1}^{i_{1}} \text { and } \gamma^{j} \text { is } A_{2}^{i_{2}} \text { and } \sigma^{j} \text { is } A_{3}^{i_{3}} \text { then } P_{g}^{j} \text { is } g^{i_{1} i_{2} i_{3}}, i_{1}=1 \ldots m_{1}, i_{2}=1 \ldots m_{2} \text {, and } i_{3}=1 \ldots m_{3} \text {. }
$$

where $m_{1}, m_{2}$, and $m_{3}$ are the number of fuzzy sets utilized to define the inputs of the fuzzy controller $P_{s}^{j}, \gamma^{j}$, and $\sigma^{j}$, respectively. Let $x_{a}$ be the variable of the $a$-th input, $\mu_{a}^{i_{a}}\left(x_{a}\right)$ be the $i_{a}-$ th membership functions of the $a$-th fuzzy input, $a=1 \ldots 3$. The membership function $\mu_{a}^{i_{a}}\left(x_{a}\right)$ is defined as a symmetric orthogonal triangular function:

$$
\mu_{a}^{i_{a}}\left(x_{a}\right)=\left\{\begin{array}{cc}
\left(x_{a}-d_{a}^{i_{a}-1}\right) /\left(d_{a}^{i_{a}}-d_{a}^{i_{a}-1}\right), & \text { if } d_{a}^{i_{a}-1} \leq x_{a}<d_{a}^{i_{a}} \\
\left(d_{a}^{i_{a}+1}-x_{a}\right) /\left(d_{a}^{i_{a}+1}-d_{a}^{i_{a}}\right), & \text { if } d_{a}^{i_{a}} \leq x_{a}<d_{a}^{i_{a}+1} \\
0, & \text { otherwise }
\end{array}\right.
$$

where $d_{a}^{i_{a}}$ is the center of the triangular membership function associated with the $i_{a}$ - th membership function. Note that the first and $m_{a}-t h$ membership functions are defined as half-symmetric triangular functions, i.e.,

$$
\begin{aligned}
& \mu_{a}^{1}\left(x_{a}\right)=\left\{\begin{array}{cc}
\left(d_{a}^{1}-x_{a}\right) /\left(d_{a}^{1}\right), & \text { if } 0 \leq x_{a} \leq d_{a}^{1} \\
0, & \text { otherwise. }
\end{array}\right. \\
& \mu_{a}^{m_{a}}\left(x_{a}\right)=\left\{\begin{array}{c}
\left(x_{a}-d_{a}^{m_{a}-1}\right) /\left(d_{a}^{m_{a}}-d_{a}^{m_{a}-1}\right), \text { if } d_{a}^{m_{a}-1} \leq x_{a} \leq d_{a}^{m_{a}} \\
0, \quad \text { otherwise. }
\end{array}\right.
\end{aligned}
$$

The universe of discourse of all membership functions in Equations (6)-(8) are normalized in the range of $[-1,1]$. It results in all centers of the triangular membership functions $d_{a}^{i_{a}}(\cdot) \in[-1,1], a=1 \ldots 3$. The output at the $j$-th time step of the fuzzy controller in (5) is calculated as follows by using product inference and the center average defuzzification approach:

$$
P_{g}^{j}=\frac{\sum_{i_{1}=1}^{m_{1}} \sum_{i_{2}=1}^{m_{2}} \sum_{i_{3}=1}^{m_{3}} g^{i_{1}, i_{2}, i_{3}} \prod_{a=1}^{3} \mu_{a}^{i_{a}}\left(x_{a}\right)}{\sum_{i_{1}=1}^{m_{1}} \sum_{i_{2}=1}^{m_{2}} \sum_{i_{3}=1}^{m_{3}} \prod_{a=1}^{3} \mu_{a}^{i_{a}}\left(x_{a}\right)} .
$$

Only two adjacent orthogonal membership functions are activated due to any input signal. Assume that $i_{a}^{*}-t h$ and $\left(i_{a}^{*}+1\right)-t h$ are activated, $a=1 \ldots 3$, due to inputs $P_{s}^{j}=x_{1}^{*}, \gamma^{j}=x_{2}^{*}$, and $\sigma^{j}=x_{3}^{*}$ at the $j$-th time step. Then,

$$
\sum_{i_{1}=1}^{m_{1}} \sum_{i_{2}=1}^{m_{2}} \sum_{i_{3}=1}^{m_{3}} \prod_{a=1}^{3} \mu_{a}^{i_{a}}\left(x_{a}^{*}\right)=\sum_{i_{1}=i_{1}^{*}}^{i_{1}^{*}+1} \sum_{i_{2}=i_{2}^{*}}^{i_{2}^{*}+1} \sum_{i_{3}=i_{3}^{*}}^{i_{3}^{*}+1} \prod_{a=1}^{3} \mu_{a}^{i_{a}}\left(x_{a}^{*}\right) .
$$

It is obvious for the orthogonal membership function that:

$$
\mu_{a}^{i_{a}+1}\left(x_{a}\right)=1-\mu_{a}\left(x_{a}\right) .
$$

Therefore,

$$
\sum_{i_{1}=1}^{m_{1}} \sum_{i_{2}=1}^{m_{2}} \sum_{i_{3}=1}^{m_{3}} \prod_{a=1}^{3} \mu_{a}^{i_{a}}\left(x_{a}^{*}\right)=\prod_{a=1}^{3}\left(\mu_{a}^{i_{a}^{*}}\left(x_{a}^{*}\right)+\left(1-\mu_{a}^{i_{a}^{*}}\left(x_{a}^{*}\right)\right)\right)=1 .
$$


Substituting (12) into (9) yields:

$$
P_{g}^{j}=\sum_{i_{1}=i_{1}^{*}}^{i_{1}^{*}+1} \sum_{i_{2}=i_{2}^{*}}^{i_{2}^{*}+1} \sum_{i_{3}=i_{3}^{*}}^{i_{3}^{*}+1} g^{i_{1}^{*} i_{2}^{*} i_{2}^{*} \prod_{3}^{*}} \prod_{a=1}^{3} \mu_{a}^{i_{a}^{*}}\left(x_{a}^{*}\right)
$$

for any input $P_{s}^{j}=x_{1}^{*}, \gamma^{j}=x_{2}^{*}$ and $\sigma^{j}=x_{3}^{*}$ at the $j$-th time step. The calculation of defuzzification can be greatly simplified as in Equation (13).

\subsection{Fuzzy Rule Base}

There are totally $\left(m_{1} \times m_{2} \times m_{3}\right)$ fuzzy rules in the fuzzy rule base as shown in (5). A self-learning scheme is proposed in this paper to learn parameters of every fuzzy rule in the fuzzy rule base. It is obvious that considerable computation effort is necessary to learn the entire fuzzy rule base in (5). The MTMFs are utilized so that the number of parameters required to be learned for the fuzzy rule base can be greatly reduced.

The centers of the $i_{a}$-th MTMF for the $a$-th input denoted as $\underline{d}_{a}^{i_{a}}$ are defined to be uniformly distributed in $[0,1]$ such that

$$
\underline{d}_{a}^{i_{a}}=i_{a} /\left(m_{a}-1\right), i_{a}=0,1, \ldots,\left(m_{a}-1\right), a=1 \ldots 3 .
$$

Without loss of generality, let $m_{\mathfrak{a}}$ be an odd number. Denote the modulation factor $\theta_{a}$ such that $0<\theta_{a}<1$ and the modulation exponent as $\eta_{a}$ such that:

$$
\eta_{a}=\left(m_{a}-1\right) / 2-\left|i_{a}-\left(m_{a}-1\right) / 2\right|, i_{a}=0,1, \ldots,\left(m_{a}-1\right) .
$$

The centers of the MTMF defined in (14) are modulated using a modulation factor $\theta_{a}$ and the exponent $\eta_{a}$ in (15) as follows:

$$
\hat{d}_{a}^{i_{a}}=\underline{d}_{a}^{i_{a}}\left(1+\theta_{a}\right)^{\eta_{a}}, i_{a}=0,1, \ldots,\left(m_{a}-1\right) .
$$

The centers of the MTMF can be added an offset factor $0<\alpha_{a}<1$ except for the first one that is set at the origin. Therefore, the centers of the MTMF are defined as follows:

$$
d_{a}^{i_{a}}=\left(1-\alpha_{a}\right) \hat{d}_{a}^{i_{a}}+\alpha_{a}, i_{a}=1 \ldots\left(m_{a}-1\right), a=1 \ldots 3 .
$$

Referring to Equations (14)-(17), the fuzzy rule base with a total of $\left(m_{1} \times m_{2} \times m_{3}\right)$ fuzzy rules can be defined with only six parameters, that is, modulation factor $\theta_{a}$ and offset factor $\alpha_{a}, a=1 \ldots 3$. Note that the fuzzy rule base with the fuzzy sets defined by the MTMF is valid only if the centers of the MTMF defined in Equation (17) are in a strictly increasing sequence. The centers of the MTMF defined in Equation (17) form a strictly increasing sequence if the following theorem is satisfied.

Theorem 1. The centers of the MTMF defined in (17) are in a strictly increasing sequence if $0<\theta_{a} \leq 2 /\left(m_{a}-1\right)$.

Proof. Referring to (15), the range of $i_{a}$ can divided into two parts. If $i_{a} \leq\left(m_{a}-1\right) / 2, \eta_{a}=i_{a}$. Then,

$$
\begin{gathered}
\hat{d}_{a}^{i_{a}+1}-\hat{d}_{a}^{i_{a}}=\frac{i_{a}+1}{m_{a}-1}\left(1-\theta_{a}\right)^{i_{a}+1}-\frac{i_{a}}{m_{a}-1}\left(1-\theta_{a}\right)^{i_{a}} \\
=\frac{\left(1-\theta_{a}\right)^{i_{a}}\left(1-\theta_{a}-i_{a} \theta_{a}\right)}{m_{a}-1} .
\end{gathered}
$$

Since $0<\theta_{a}<1$, it is shown in Equation (18) that $\hat{d}_{a}^{i_{a}+1}>\hat{d}_{a}^{i_{a}}$ if

$$
\theta_{a}<1 /\left(1+i_{a}\right)
$$


The condition in Equation (19) is satisfied for both cases $i_{a} \leq\left(m_{a}-1\right) / 2$ and $i_{a}+1 \leq\left(m_{a}-1\right) / 2$. The intersection of both cases is $i_{a} \leq\left(m_{a}-3\right) / 2$. Therefore, the range of $\theta_{a}$ can be determined by substituting $i_{a}=\left(m_{a}-3\right) / 2$ into Equation (19):

$$
\theta_{a} \leq 2 /\left(m_{a}-1\right)
$$

Consider the other part of $i_{a}$, that is, $i_{a}>\left(m_{a}-1\right) / 2$. As $i_{a}$ is an integer, this is equivalent to the condition $i_{a} \geq\left(m_{a}-1\right) / 2+1=\left(m_{a}+1\right) / 2$. Referring to Equation (15), $\eta_{a}=m_{a}-i_{a}-1$ if $i_{a} \geq\left(m_{a}+1\right) / 2$. Then:

$$
\begin{gathered}
\hat{d}_{a}^{i_{a}+1}-\hat{d}_{a}^{i_{a}}=\frac{i_{a}+1}{m_{a}-1}\left(1-\theta_{a}\right)^{m_{a}-i_{a}-2}-\frac{i_{a}}{m_{a}-1}\left(1-\theta_{a}\right)^{m_{a}-i_{a}-1} \\
=\frac{\left(1+i_{a} \theta_{a}\right)\left(1-\theta_{a}\right)^{m_{a}-i_{a}-2}}{m_{a}-1} .
\end{gathered}
$$

Referring to Equation (21), it is obvious that $\hat{d}_{a}^{i_{a}+1}>\hat{d}_{a}^{i_{a}}$ for $i_{a} \geq\left(m_{a}+1\right) / 2$ because $0<\theta_{a}<1$. The above analysis reveals that the sequence $\hat{d}_{a}^{i_{a}}, i_{a}=1 \ldots\left(m_{a}-1\right)$, is a strictly increasing sequence if $0<\theta_{a} \leq 2 /\left(m_{a}-1\right)$. Referring to Equation (17),

$$
d_{a}^{i_{a}+1}-d_{a}^{i_{a}}=\left(1-\alpha_{a}\right)\left(\hat{d}_{a}^{i_{a}+1}-\hat{d}_{a}^{i_{a}}\right) .
$$

Since the offset is defined in the range of $0<\alpha_{a}<1, d_{a}^{i_{a}+1}-d_{a}^{i_{a}}>0$ if $\hat{d}_{a}^{i_{a}+1}-\hat{d}_{a}^{i_{a}}>0$. Therefore, the sequence of MTMF centers $d_{a}^{i_{a}}, i_{a}=1 \ldots\left(m_{a}-1\right)$, is also an increasing sequence with the constraint $0<\theta_{a} \leq 2 /\left(m_{a}-1\right)$.

The MTMF with $m_{a}=11$ for both cases: $\theta_{a}=0.1$ and $\alpha_{a}=0$, and $\theta_{a}=0.16$ and $\alpha_{a}=0.2$, are compared in Figures $1 \mathbf{b}$ and $2 \mathrm{a}$, respectively. The antecedent part of the fuzzy rule base with $\left(m_{1} \times m_{2} \times m_{3}\right)$ fuzzy rules in Equation (5) are described by MTMF using only six parameters $\theta_{a}$ and $\alpha_{a}, a=1 \ldots 3$. Howevser, there are still $\left(m_{1} \times m_{2} \times m_{3}\right)$ fuzzy singletons in the consequent part of the fuzzy rule base. To further reduce the number of parameters to be learned, an effective parameterization scheme is adopted for the fuzzy singletons in the consequent part without reducing accuracy to a great extent. For the convenience of representation, the fuzzy sets of the inputs are arranged from small values to medium values and then to large values in every table of fuzzy rules.

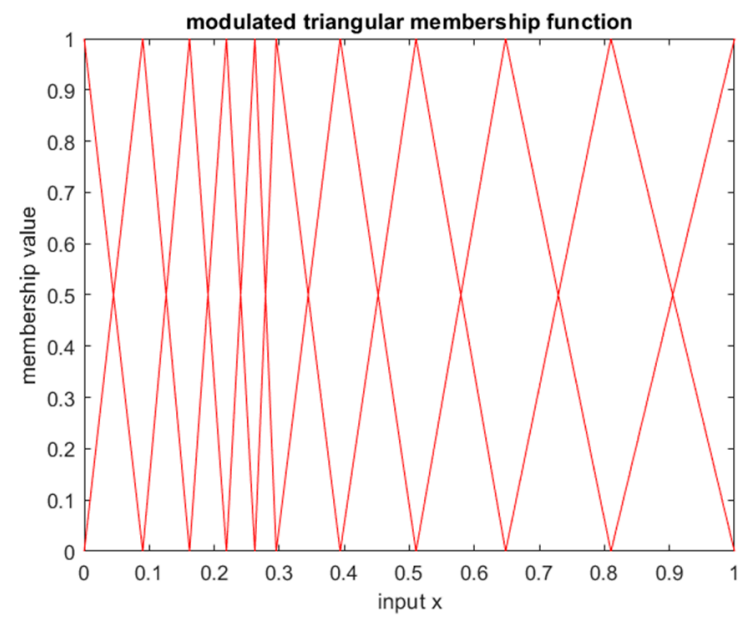

(a)

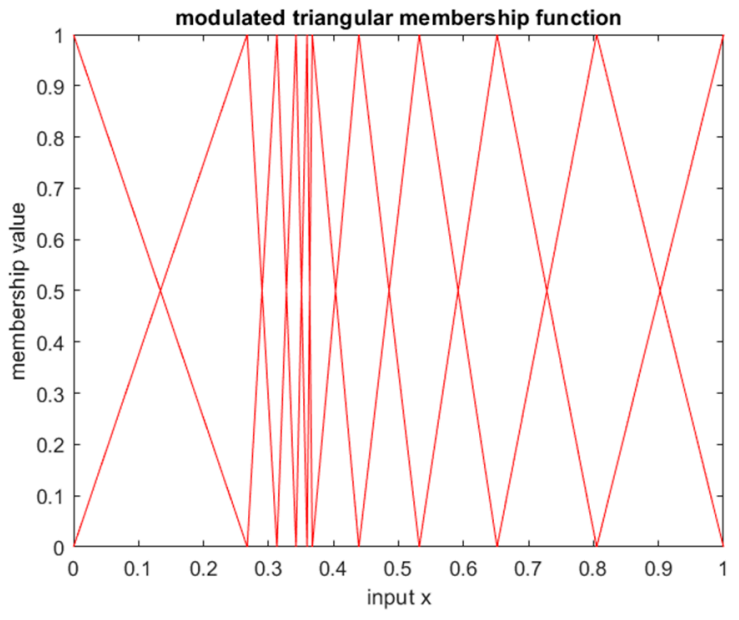

(b)

Figure 2. Illustration of modulated triangular membership functions with $m_{a}=11$ and (a) $\theta_{a}=0.1$, $\alpha_{a}=0 ;(\mathbf{b}) \theta_{a}=0.16, \alpha_{a}=0.2$. 
The design of the consequent part of the fuzzy controller aims to reduce electricity cost. Referring to the characteristics of the HEMS and the load balance equation, the design of the consequent part of fuzzy controller is mainly based on following considerations.

1. The fuzzy controller should allow more rectified power $P_{g}^{j}$ into DC bus from the grid as the net load difference $P_{s}^{j}$ increases because increase in $P_{s}^{j}$ implies either that the net load demand increases or that the solar energy decreases.

2. The fuzzy controller should allow less rectified power $P_{g}^{j}$ into DC bus as ESS has a higher amount of stored energy, i.e., as the SOC of the ESS $\gamma^{j}$ increases. Using stored energy in the ESS in a smart way could result in reducing electricity cost.

3. The fuzzy controller should allow less rectified power $P_{g}^{j}$ into DC bus as the RTP increases because the main function of this fuzzy controller is to reduce the total electricity cost paid to the utility.

Assume that the increasing or decreasing rates of the fuzzy output with respect to every $a$-th fuzzy input are divided into two parts with $\beta_{a}$ being the intermediate value, $a=1$...3. Without loss of generality, let the number of membership functions corresponding to every fuzzy input be an odd number. The intermediate value $\beta_{a}$ is set to be the center of the MTMF numbered as $\left(m_{a}-1\right) / 2$. The first and second part of the increasing or decreasing rate $\lambda_{a 1}$ and $\lambda_{a 2}$ are respectively defined as:

$$
\begin{gathered}
\lambda_{a 1}=\frac{2 \beta_{a}}{m_{a}-1}, \\
\lambda_{a 2}=\frac{2\left(1-\beta_{a}\right)}{m_{a}-1} .
\end{gathered}
$$

Note that $\beta_{1} \in(0,1)$ and $\beta_{2}, \beta_{3} \in(-1,0)$. Let $\lambda_{a}^{i_{a}}$ be the increasing or decreasing rate for the $i_{a}-$ th MTMF associated with the $a$-th input, $a=1 \ldots 3$. The normalized fuzzy singletons $g^{i_{1} i_{2} i_{3}}$ in the consequent parts of all $\left(m_{1} \times m_{2} \times m_{3}\right)$ fuzzy rules in (5) are defined as:

$$
g^{i_{1} i_{2} i_{3}}=i_{1} \lambda_{1}^{i_{1}}-i_{2} \lambda_{2}^{i_{2}}-i_{3} \lambda_{3}^{i_{3}}
$$

where:

$$
\lambda_{a}^{i_{a}}=\left\{\begin{array}{lc}
\lambda_{a 1}, & \text { if } i_{a} \leq\left(m_{a}-1\right) / 2 \\
\lambda_{a 2}, & \text { otherwise. }
\end{array}\right.
$$

Referring to Equations (23)-(26), the consequent part of the entire fuzzy rule base is defined by only three parameters $\beta_{1}, \beta_{2}$, and $\beta_{3}$. The singleton at the consequent part of every fuzzy rule is determined by the increasing or decreasing rate as shown in Equation (25). The increasing or decreasing rate is assigned two different values depending on whether the associated MTMF is in the first or second half of all $m_{a}$ membership functions as shown in Equation (26). Therefore, both the antecedent and consequent parts of the entire rule base are determined only by nine parameters $\left(\theta_{a}, \alpha_{a}, \beta_{a}\right), a=1 \ldots 3$.

\section{Self-Learning of Fuzzy Controllers}

As the HEMS is initially installed and operated with the initial parameter settings for a certain period of time, the fuzzy controller that controls the rectifier output can be optimized or updated on the basis of the recorded historical data. The previously optimized fuzzy controller has to be updated from time to time when the environmental settings or home load variations are updated. For the fuzzy controller learning, assume that the profiles $P_{d^{\prime}}^{j} P_{P V^{\prime}}^{j} \gamma^{j}$, and $\sigma^{j}$ in the previous $D$ days are recorded. Given that there are $J$ time steps in every individual day, the profiles of totally $L(=D \times J)$ time steps are recorded for self-learning of the fuzzy controller $\mathbb{F}\left(P_{s}^{j}, \gamma^{j}, \sigma^{j}\right)$ in (2). 
The GA is applied to learn the parameters $\left(\theta_{a}, \alpha_{a}, \beta_{a}\right), a=1 \ldots 3$, that parameterize the entire fuzzy rule base. Suppose that $G$ chromosomes are generated in the gene pool of the GA. By denoting the parameters encoded in the $i$-th chromosome of the $k$-th generation as $\rho(i, k)$, then:

$$
\rho(i, k) \equiv\left\{\left(\theta_{a}(i, k), \alpha_{a}(i, k), \beta_{a}(i, k)\right) \mid a=1 \ldots 3\right\}, i=1 \ldots G .
$$

The output of the fuzzy controller at every $j$-th time step parameterized by $\rho(i, k)$ is defined as:

$$
P_{g}^{j}(i, k)=\left.\mathbb{F}\left(P_{s}^{j}, \gamma^{j}, \sigma^{j}\right)\right|_{\rho(i, k)}, j=1 \ldots L .
$$

Let $H(i, k)$ be the fitness function corresponding to $\rho(i, k)$ and $n_{d}$ be the number of sampling intervals in $1 \mathrm{~h} . H(i, k)$ can be defined as the electricity cost associated with $P_{g}^{j}(i, k)$ in Equation (28) based on the profiles recorded in previous $L$ time steps:

$$
H(i, k)=\sum_{j=1}^{L}\left(\frac{P_{g}^{j}(i, k) \sigma^{j}}{n_{d}}+v\left(\gamma^{j}\right)\right)
$$

where $v\left(\gamma^{j}\right)$ is the penalty function that prevents the ESS from over discharging. Denote $\chi$ as the lower bound of the SOC that allows the ESS to discharge. Define $v\left(\gamma^{j}\right)$ as:

$$
v\left(\gamma^{j}\right)=\left\{\begin{array}{c}
0, \text { if } \gamma^{j}<\chi \\
\omega>>0, \text { otherwise }
\end{array}\right.
$$

where $\omega$ is a large number. Let $\hat{\rho}(k)$ be the best parameters searched in the $k$-th generation and is obtained by searching all $G$ chromosomes in the gene pool of the $k$-th generation by minimizing $H(i, k)$ defined in Equation (29). Therefore:

$$
\hat{\boldsymbol{\rho}}(k)=\underset{\rho(i, k), i=1 \ldots G}{\operatorname{Argmin}}(H(i, k)) .
$$

Referring to (20), GA's search range of $\theta_{a}^{i}$ can be limited to $\theta_{a}^{i} \in\left(0,2 /\left(m_{a}-1\right)\right], a=1 \ldots 3$. The search range of $\alpha_{a}^{i}$ and $\beta_{a}^{i}$ are given as $\alpha_{a}^{i} \in(0,1), a=1 \ldots 3$; and $\beta_{1}^{i} \in(0,1), \beta_{2}^{i}, \beta_{3}^{i} \in(-1,0)$. The crossover operation is conducted parameter by parameter for all nine parameters in every chromosome. Denote $\mathfrak{R}_{G \backslash i}$ as an operator generating a random integer in the range of $[1, G]$ but different from the operand $i$. Without loss of generality, take $\theta_{a}$ as an example:

$$
\theta_{a}(i, k+1)=\xi_{1} \theta_{a}(i, k)+\left(1-\xi_{1}\right) \theta_{a}\left(\mathfrak{R}_{G \backslash i}, k\right)
$$

where $\xi_{1} \in(0,1)$ is a random real number. Every chromosome is selected for mutation with probability $\beta$. The mutation is conducted by randomly perturbing every parameter in the chromosome within a given range. Without loss of generality, let $\theta_{a}$ in the $j$-th chromosome be selected for mutation, then:

$$
\theta_{a}(j, k+1)=\theta_{a}(j, k)+\xi_{2} w_{\theta_{a}}
$$

where $\xi_{2} \in(-1,1)$ is a random real number, and $w_{\theta}$ is a constant denoting the perturbation range for mutation. If the mutated parameter is out of the searching range, then the mutation is repeated until the mutated result is within the allowed range. The elitist scheme is applied to the proposed GA, i.e., the best chromosome associated with the minimum fitness value in every generation is passed to the next generation. Assume that it takes $N_{g}$ generations for the GA to converge, the best parameter $\rho^{*}$ for the fuzzy rule base is the best chromosome obtained in the $N_{g}-$ th generation, i.e., $\rho^{*}=\hat{\rho}\left(N_{g}\right)$. 


\section{Experiments}

An environment practically simulating a smart home with a home power system controlled by the HEMS is developed and used to test the effectiveness and efficiency of the proposed self-learning fuzzy controller. The appliances and their associated power ratings in the simulated smart home are shown in Table 1. The rated output power of the PV panel is $4 \mathrm{~kW}$. The sampling interval is set as $5 \mathrm{~min}$. The self-learning fuzzy controller is automatically learned based on the 14 days of previously recorded load profiles. Referring to Equation (29), the total number of time steps for evaluation $L$ are 4032. The fuzzy controller was initially set in the HEMS to control the grid power $P_{g}$ to the home power system. After collecting 14 days of load profiles, the fuzzy controller parametrized by the MTMF was optimized through self-learning. The performance of the optimized fuzzy controller on both sunny and cloudy days is shown in Figures 2 and 3, respectively.

Table 1. Consumptions of Tested Appliances.

\begin{tabular}{cccc}
\hline Appliance & Power $\mathbf{( k W )}$ & Appliance & Power $\mathbf{( k W )}$ \\
\hline clothes dryer & $0.3-0.8$ & rice cooker & 0.6 \\
water pump & 0.74 & lighting & $0.22 \times 3$ \\
refrigerator & $0.2-0.85$ & dish washer & $0.2-0.6$ \\
\hline
\end{tabular}

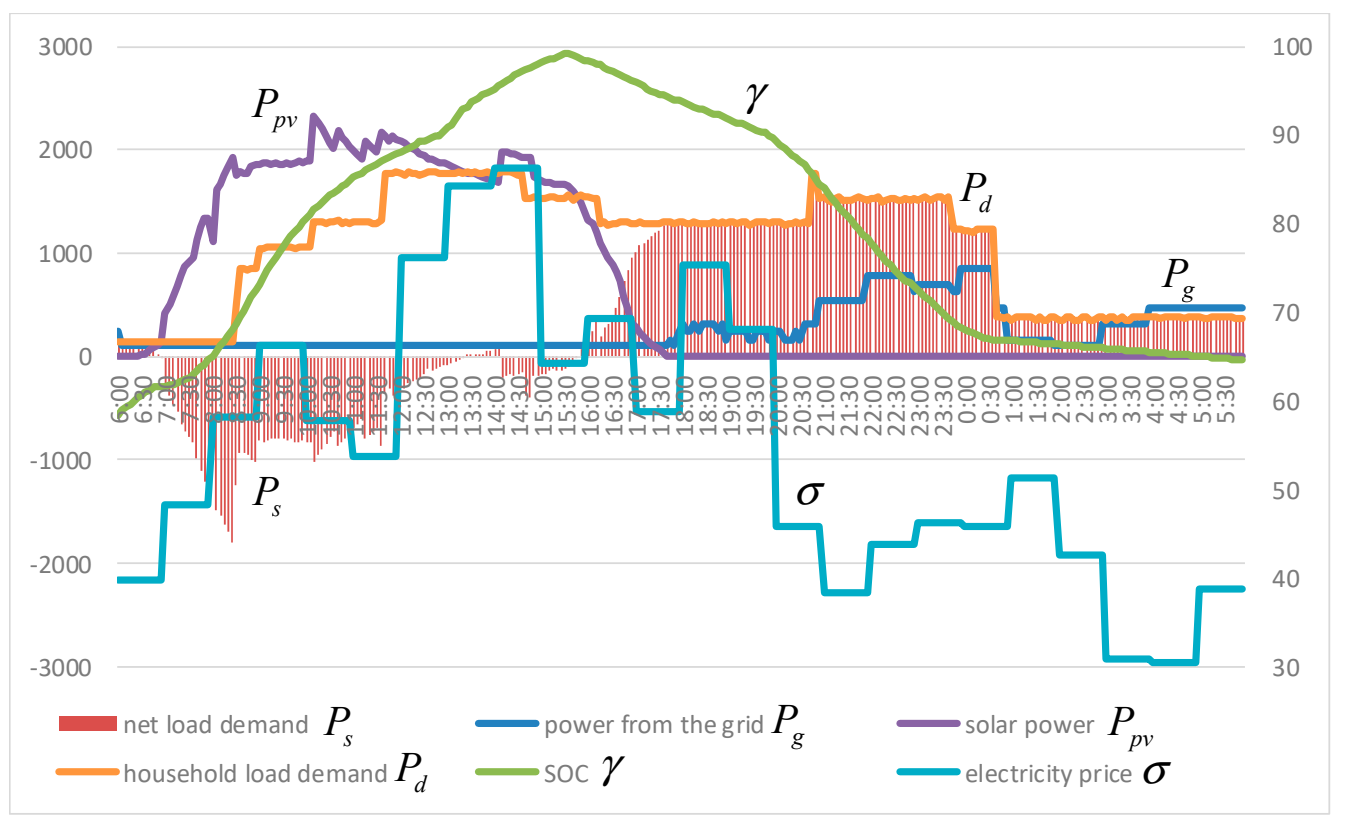

Figure 3. Output of fuzzy controller for the first scenario (sunny day).

The charging and discharging efficiency $b_{c}$ and $b_{d}$, respectively, associated with the efficiency constant $\eta$ in (4) are both set as 0.95 . The lower bound $\chi$ of the SOC for the ESS is 0.6. The number of membership functions for the fuzzy controller $m_{a}=11, a=1 \ldots 3$. For the GA, the number of chromosomes in the gene pool $G=50$, and the mutation probability $\beta=0.02$. Moreover, the perturbation range for $\theta_{a}$, i.e., $w_{\theta_{a}}=0.02$ in (33), the perturbation ranges for $\alpha_{a}$ and $\beta_{a}$, i.e., $w_{\alpha_{a}}$ and $w_{\beta_{a}}$ are both set as $w_{\alpha_{a}}=w_{\beta_{a}}=0.1$.

The first scenario of the experiment was to test the performance of the proposed fuzzy controller with the HEMS on a sunny day. The average theoretical output power of the PV panels $\bar{P}_{t h}=1868$ W. It is shown in Figure 3 that the solar power $P_{p v}$ was greater than the household load demand $P_{d}$ from 7:00 to 16:00, thus leading to negative net load demand $P_{s}\left(=P_{d}-P_{p v}\right)$ within this period. The additional solar power can also charge the EES so that the SOC $\gamma$ increased before 16:00. 
No additional power from the grid was required during this period. Therefore, the fuzzy controller allowed almost no power from the grid $P_{g}$. Although the net load demand $P_{s}$ became positive, both the SOC $\gamma$ and the RTP $\sigma$ were still high from 16:00 to 17:30. $P_{g}$ was controlled to be approximately 0 during this period. Since the RTP $\sigma$ received at every time step was still high from 17:30 to 20:00, the fuzzy controller controlled $P_{g}$ to a reasonably small value so that the stored energy in the ESS can be used as much as possible. The RTP $\sigma$ dropped to reasonably small values after 20:00. The $P_{g}$ controlled by the fuzzy controller increased to reasonably larger values, thus providing sufficient power to cope with net load demand. The net load demand significantly reduced to much smaller values after 01:00, and $P_{g}$ was controlled to be sufficiently large enough providing the required electricity because the RTP $\sigma$ were comparatively low during this period.

The second scenario of the experiment was to test the performance of the proposed fuzzy controller with the HEMS on a cloudy day. Referring to Figure 4, the solar power was not sufficient to provide the net load demand on a cloudy day. The net load demand $P_{S}$ varied from 6:00 to 15:00 due to insufficient solar power during this period. By integrating the ESS and solar power, the fuzzy controller dynamically controlled $P_{g}$ to cope with the dynamic net load demand. On the other hand, the controller attempted to minimize the use of $P_{g}$ when the RTP $\sigma$ were high. The RTP $\sigma$ were high from 12:00 to 15:00, the fuzzy controller only allowed reasonably small amount of $P_{g}$ used in the home power system. The net load demand $P_{s}$ was high from 18:00 to 20:00. However, the fuzzy controller allowed reasonably small amount of $P_{g}$, thus leading to a higher amount of discharging power from the ESS due to the high RTP $\sigma$ during this period. The RTP reduced after 21:00, and the fuzzy controller allowed a higher amount of $P_{g}$ from the grid following the variation of net load demand $P_{s}$.

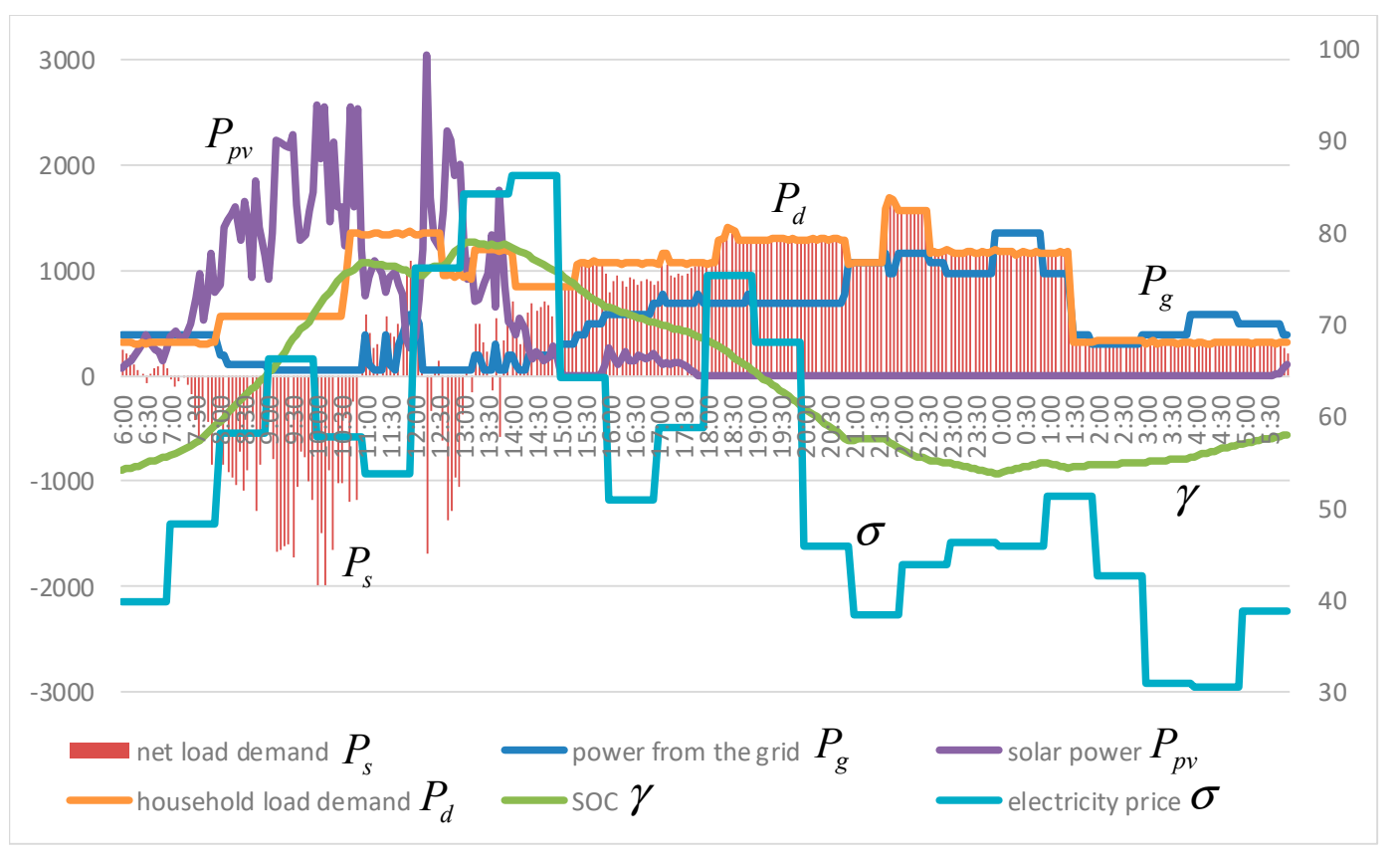

Figure 4. Output of fuzzy controller for the second scenario (cloudy day).

\section{Conclusions}

A computationally efficient self-learning fuzzy controller for the HEMS has been proposed for reducing the electric energy cost for smart homes. With the parsimonious parameterization design of the fuzzy rule base, the fuzzy controller can efficiently regulate AC power from the grid. Moreover, the fuzzy controller only requires six and three parameters to automatically learn the antecedent and consequent part of the entire fuzzy rule base, respectively, for the three-input/one-output fuzzy controller. The proposed design of the fuzzy controller for the HEMS has a good commercialization potential because it was intentionally designed to fit the embedded system by using low computational 
power CPUs. As long as the power balance equation in Equation (1) is satisfied, the same control scheme of the proposed fuzzy controller can be applied to a smart home with more number of integrated energy sources such as fuel cells or electric vehicle charging and discharging.

Moreover, the problem to be solved in this paper is a system with varying conditions. More uncertainty quantification methodologies can be applied to this problem. The self-learning fuzzy controller proposed in this paper can be classified as a non-probabilistic approach solving the forward uncertainty propagation problem. Other approaches for the forward uncertainty propagation problem such as the one in this paper are worth investigating.

Author Contributions: Conceptualization, L.Y.; methodology, L.Y.; software, F.H.H. and C.-C.L.; validation, L.Y. and C.-C.L.; formal analysis, L.Y.; investigation L.Y. and F.H.H.; resources, L.Y.; data curation, F.H.H. and C.-C.L.; writing, L.Y.; supervision, L.Y.; project administration, L.Y.; funding acquisition, L.Y. All authors have read and agreed to the published version of the manuscript.

Funding: This research was funded by Ministry of Science and Technology, Taiwan, grant number MOST 107-2221-E-027-086-MY3.

Conflicts of Interest: The authors declare no conflict of interest.

\section{References}

1. Shareef, H.; Ahmed, M.S.; Mohamed, A.; Al Hassan, E. Review on home energy management system considering demand responses, smart technologies, and intelligent controllers. IEEE Access 2018, 6, 24498-24509. [CrossRef]

2. Rastegar, M.; Fotuhi-Firuzabad, M.; Aminifar, F. Load commitment in a smart home. Appl. Energy 2012, 96, 45-54. [CrossRef]

3. Mohsenian-Rad, A.; Leon-Garcia, A. Optimal residential load control with price prediction in real-time electricity pricing environments. IEEE Trans. Smart Grid 2010, 1, 120-133. [CrossRef]

4. Nanda, A.K.; Panigrahi, C.K. Review on smart home energy management. Int. J. Ambient Energy 2016, 37, 541-546. [CrossRef]

5. Joo, I.-Y.; Choi, D.-H. Distributed optimization framework for energy management of multiple smart homes with distributed energy resources. IEEE Access 2017, 5, 15551-15560. [CrossRef]

6. Huang, Y.; Wang, L.; Guo, W.; Kang, Q.; Wu, Q. Chance constrained optimization in a home energy management system. IEEE Trans. Smart Grid 2018, 9, 252-260. [CrossRef]

7. Ozturk, Y.; Senthilkumar, D.; Kumar, S.; Lee, G. An intelligent home energy management system to improve demand response. IEEE Trans. Smart Grid 2013, 4, 694-701. [CrossRef]

8. Basit, A.; Sidhu, G.A.S.; Mahmood, A.; Gao, F. Efficient and autonomous energy management techniques for the future smart homes. IEEE Trans. Smart Grid 2017, 8, 917-926. [CrossRef]

9. Luo, F.; Ranzi, g.; Wan, C.; Xu, Z.; Dong, Z.Y. A multistage home energy management system with residential photovoltaic penetration. IEEE Trans. Ind. Inform. 2019, 15, 116-126. [CrossRef]

10. Nguyen, H.T.; Nguyen, D.T.; Le, B. Energy management for households with solar assisted thermal load considering renewable energy and price uncertainty. IEEE Trans. Smart Grid 2015, 6, 301-314. [CrossRef]

11. Wang, Z.; Gu, C.; Li, F.; Bale, P.; Sun, H. Active demand response using shared energy storage for household energy management. IEEE Trans. Smart Grid 2013, 4, 1888-1897. [CrossRef]

12. Li, T.; Dong, M. Real-time residential-side joint energy storage management and load scheduling with renewable integration. IEEE Trans. Smart Grid 2018, 9, 283-298. [CrossRef]

13. Borenstein, S.; Jaske, M.; Rosenfeld, A. Dynamic Pricing, Advanced Metering and Demand Response in Electricity Markets; UC Berkeley, Center for the Study of Energy Markets: Berkeley, CA, USA, 2002.

14. Jinsiwale, R.; Divan, D. Decentralized real-time pricing to achieve integrated transactive and physical grids. IEEE Access 2019, 9, 132525-132541. [CrossRef]

15. Roozbehani, M.; Dahleh, M.A.; Mitter, S.K. Volatility of power grids under real-time pricing. IEEE Trans. Power Syst. 2012, 27, 1926-1940. [CrossRef]

16. Liu, H.; Shi, J. Applying ARMA-GARCH approaches to forecasting short-term electricity prices. Energy Econ. 2013, 37, 152-166. [CrossRef] 
17. Papadimitriou, T.; Gogas, P.; Stathakis, E. Forecasting energy markets using support vector machines. Energy Econ. 2014, 44, 135-142. [CrossRef]

18. Cerjan, M.; Matijas, M.; Delimar, M. Dynamic hybrid model for short-term electricity price forecasting. Energies 2014, 7, 3304-3318. [CrossRef]

19. Alamaniotis, M.; Bargiotas, D.; Bourbakis, N.G.; Tsoukalas, L.H. Genetic optimal regression of relevance vector machines for electricity pricing signal forecasting in smart grids. IEEE Trans. Smart Grid 2018, 6, 2997-3005. [CrossRef]

20. Hansen, T.; Chong, E.; Suryanarayanan, S.; Maciejewski, A.; Siegel, H. A partially observable Markov decision process approach to residential home energy management. IEEE Trans. Smart Grid 2018, 9, 1271-1281. [CrossRef]

21. Vivekananthan, C.; Mishra, Y.; Li, F. Real-time price based home energy management scheduler. IEEE Trans. Power Syst. 2015, 30, 2149-2159. [CrossRef]

22. Muratori, M.; Rizzoni, G. Residential demand response: Dynamic energy management and time-varying electricity pricing. IEEE Trans. Power Syst. 2016, 31, 1108-1117. [CrossRef]

23. Hubert, T. Grijalva Modeling for residential electricity optimization in dynamic pricing environments. IEEE Trans. Smart Grid 2012, 3, 2224-2231. [CrossRef]

24. Althaher, S.; Mancarella, P.; Mutale, J. Automated demand response from home energy mnagement system under dynamic pricing and power and comfort constraints. IEEE Trans. Smart Grid 2015, 6, 1874-1883. [CrossRef]

25. Pilloni, V.; Floris, A.; Meloni, A.; Atzori, L. Smart home energy management including renewable sources: A QoE-driven approach. IEEE Trans. Smart Grid 2018, 9, 2006-2018. [CrossRef]

26. Khalidi, A.; Javaid, N.; Guizani, M.; Alhussein, M.; Aurangzeb, K.; Ilahi, M. Towards dynamic coordination among home appliances using multi-objective energy optimization for demand side management in smart buildings. IEEE Access 2018, 6, 19509-19529. [CrossRef]

27. Yoon, J.H.; Baldick, R.; Novoselac, A. Dynamic demand response controller based on real-time retail price for residential buildings. IEEE Trans. Smart Grid 2014, 5, 121-129. [CrossRef]

28. Yu, L.; Jiang, T.; Zou, Y. Online energy management for a sustainable smart home with an HVAC load and random occupancy. IEEE Trans. Smart Grid 2019, 10, 1646-1659. [CrossRef]

29. Zhang, D.; Li, S.; Sun, M.; O’Neill, Z. An optimal and learning-based demand response and home energy management system. IEEE Trans. Smart Grid 2016, 7, 1790-1801. [CrossRef]

30. Wu, Z.; Zhang, X.P.; Brandt, J.; Zhou, S.Y.; Li, J.N. Three control approaches for optimized energy flow with home energy management system. IEEE Power Energy Technol. Syst. J. 2015, 2, 21-31.

31. Keerthisinghe, C.; Verbič, G.; Chapman, A.C. A fast technique for smart home management: ADP with temporal difference learning. IEEE Trans. Smart Grid 2018, 9, 3291-3303. [CrossRef]

32. Li, S.; Yang, J.; Song, W.; Chen, A. A real-time electricity scheduling for residential home energy management. IEEE Int. Things J. 2019, 6, 2602-2611. [CrossRef]

33. Zhao, Z.; Lee, W.C.; Shin, Y.; Song, K.-B. An optimla scheduling method for demand response in home energy management system. IEEE Trans. Smart Grid 2013, 4, 1391-1400. [CrossRef]

34. Du, Y.F.; Jiang, L.; Li, Y.; Wu, Q. A robust optimization approach for demand side scheduling considering uncertainty of manually operated appliances. IEEE Trans. Smart Grid 2018, 9, 743-755. [CrossRef]

35. Javaid, N.; Ullah, I.; Akbar, M.; Iqbal, Z.; Khan, F.A.; Alrajeh, N.; Alabed, M.S. An intelligent load management system with renewable energy integration for smart homes. IEEE Access 2017, 5, 13587-13600. [CrossRef]

36. Wu, X.; Hu, X.; Yin, X.; Moura, S.J. Stochastic optimal energy management of smart home with PEV energy storage. IEEE Trans. Smart Grid 2018, 9, 2065-2075. [CrossRef]

37. Shafie-Khah, M.; Siano, P. A stochastic home energy management system considering satisfaction cost and response fatigue. IEEE Trans. Ind. Inform. 2018, 14, 629-638. [CrossRef]

38. Tung, S.W.; Quek, C.; Guan, C. SaFIN: A self-adaptive fuzzy inference network. IEEE Trans. Neural Netw. 2011, 22, 1928-1940. [CrossRef]

39. Hsu, C.-F. Self-organizing adaptive fuzzy neural control for a class of nonlinear systems. IEEE Trans. Neural Netw. 2007, 18, 1232-1241. [CrossRef]

40. Zhou, C.; Quach, D.-C.; Xiong, N.; Huang, S.; Zhang, Q.; Yin, Q.; Vasilakos, A.V. An improved direct adaptive fuzzy controller of an uncertain PMSM for web-based E-service systems. IEEE Trans. Fuzzy Syst. 2015, 23, 58-71. [CrossRef] 
41. Fischle, K.; Schröder, D. An improved stable adaptive fuzzy control method. IEEE Trans. Fuzzy Syst. 1999, 7, 27-40. [CrossRef]

42. Dai, A.; Zhou, X.; Liu, X. Design and simulation of a genetically optimized fuzzy immune PID controller for a novel grain dryer. IEEE Access 2017, 5, 14981-14990. [CrossRef]

43. Giordano, V.; Naso, D.; Turchiano, B. Combining genetic algorithms and Lyapunov-based adaptation for online design of fuzzy controllers. IEEE Trans. Syst. Man Cybern. B Cybern. 2006, 36, 1118-1127. [CrossRef] [PubMed]

44. Boukens, M.; Boukabou, A.; Chadli, M. A real time self-tuning motion controller for mobile robot systems. IEEE CAA J. Autom. Sin. 2019, 6, 84-96. [CrossRef]

45. Juang, C.-F.; Jhan, Y.-H.; Chen, Y.-M.; Hsu, C.-M. Evolutionary wall-following hexapod robot using advanced multiobjective continuous ant colony optimized fuzzy controller. IEEE Tans. Cogn. Dev. Syst. 2018, 10, 585-594. [CrossRef]

46. Sharma, K.D.; Chatterjee, A.; Rakshit, A. A hybrid approach for design of stable adaptive fuzzy controllers employing Lyapunov theory and particle swarm optimization. IEEE Trans. Fuzzy Syst. 2009, 17, 329-342. [CrossRef]

47. Chrouta, J.; Chakchouk, W.; Zaafouri, A.; Jemli, M. Modeling and control of an irrigation station process using heterogeneous cuckoo search algorithm and fuzzy logic controller. IEEE Trans. Ind. Appl. 2019, 55, 976-990. [CrossRef]

48. Yao, L.; Huang, P.-Z. Learning of hybrid fuzzy controller for optical data storage device. IEEE ASME Trans. Mechatron. 2008, 13, 3-13. [CrossRef]

49. Yao, L.; Weng, K.-S.; Lin, T.-B. Observer based adaptive fuzzy controller with modulated membership functions for nonlinear system. Int. J. Uncertain. Fuzziness Knowl. Based Syst. 2016, 24, 137-159. [CrossRef]

50. Karnama, A.; Haghighi, E.; Vinuesa, R. Organic data centers: A sustainable solution for computing facilities. Results Eng. 2019, 4, 100063. [CrossRef]

51. Haidegger, T.; Kovács, L.; Preitl, S.; Precup, R.-E.; Benyó, B.; Benyó, Z. Controller design solutions for long distance telesurgical applications. Int. J. Artif. Intell. 2011, 6, 48-71.

52. Haidegger, T.; Kovács, L.; Preitl, S.; Precup, R.-E.; Benyó, B.; Benyó, Z. Cascade control for telerobotic systems serving space medicine. IFAC Proc. 2011, 44, 3759-3764. [CrossRef] 